\title{
ABUSO DE DROGAS: RELAÇÃO ENTRE HEPATITE C E CO-INFECÇÃO COM HIV
}

\section{Ravena Cibelle Nunes Silva}

Farmacêutica pela Universidade Federal do Ceará (UFC), Brasil.

\section{Edilson Martins Rodrigues Neto}

Farmacêutico pela Universidade Federal do Ceará (UFC); Mestre em Farmacologia pela Universidade Federal do Ceará (UFC); Docente Faculdade Católica Rainha do Sertão (FCRS), Quixadá (CE), Brasil.

E-mail: edilsonmrneto@hotmail.com

\section{Lidia Audrey Rocha Valadas Marques}

Cirurgiã Dentista pela Universidade Federal do Ceará (UFC); Especialista em Farmacologia Clínica pelo Instituto Ateneu.

\section{Mara Assef Leitão Lotif}

Cirurgiã Dentista pela Universidade Federal do Ceará (UFC); Mestranda em Odontologia pela Universidade Federal do Ceará (UFC), Brasil.

\section{Érika Saboia Guerra Diógenes}

Farmacêutica pela Universidade Federal do Ceará (UFC), Brasil.

\section{Renata de Sousa Alves}

Doutora em Farmacologia pela Universidade Federal do Ceará (UFC); Docente adjunta Dep. Analises Clínicas na Universidade Federal do Ceará (UFC), Brasil.
RESUMO: A partir do advento da terapia antirretroviral, a qualidade de vida dos pacientes HIV positivos foi melhorada significativamente, havendo uma redução nos casos de morte por doenças oportunistas. A hepatite $\mathrm{C}$ representa uma comorbidade para os pacientes soropositivos. Entre os indivíduos co-infectados HIV/HCV, destacamse os usuários de drogas por apresentarem comportamentos de risco. Assim, há a necessidade de se conhecer melhor a hepatite $\mathrm{C}$, o HIV e a co-infecção e sua relação com a toxicomania. Com essa finalidade, foi realizada a presente revisão sistemática que estudou as doenças envolvidas e o perfil dos indivíduos acometidos para a co-infecção HIV/ HCV.

PALAVRAS-CHAVE: Abuso de Drogas; Hepatite C; HIV.

\section{DRUG ABUSE: RELATIONSHIP BET WEEN HEPATITIS C AND CO-INFECTION BY HIV}

ABSTRACT: Since the discovery of anti-retroviral therapy, the life quality of HIV-infected people improved significantly, especially with regard to a decrease in mortality rates by opportunistic diseases. Hepatitis $\mathrm{C}$ is a co-morbidity for serum-positive patients. Illicit drug users are salient among people co-infected with HIV/HCV since they feature risk behaviors. Hepatitis C, HIV and co-infection should be better known, coupled to their relationship with drug abuse. A systemic review was undertaken which studied the diseases involved and the profile of the patients co-infected with HIC/HCV.

KEY WORDS: Drug Abuse; Hepatitis C; HIV.

\section{INTRODUÇÃO}

A descoberta do vírus da imunodeficiência humana (HIV), em 1981, representou um grande desafio para a saúde coletiva de todo o mundo, uma vez que a infecção pelo HIV se caracteriza por uma imunodeficiência progressiva e crônica (BARTLET, 2002).

Após o advento da terapia antirretroviral surgiu um número crescente de indivíduos co-infectados com os vírus HCV e HIV, que até então não tinha sido observado, pois a sobrevida dos pacientes HIV positivos era bastante limitada.

Os toxicomaníacos são apresentados como uma população muito vulnerável à contaminação pelo HIV e HCV. Esse fato pode ser relacionado com o uso dos instrumentos de consumo de drogas, sejam elas de administração parenteral ou não. A exposição direta ocorre no 
compartilhamento de seringas e agulhas na utilização da heroína ou de canudos e cachimbos com a cocaína e crack, pois existe a possibilidade de indução de lesão nasal/oral, por conta dos contaminantes desses produtos. Há também o risco indireto ligado aos seus efeitos sobre a libido, no qual algumas drogas aumentam o desejo sexual, levando aos atos sexuais inseguros, assim como a comercialização do sexo como meio de obtenção de drogas (ROSS; WILLIAMS, 2001).

O objetivo desse estudo foi realizar uma revisão sistemática sobre a associação entre a co-infecção entre o vírus HIV, hepatite $\mathrm{C}$ e uso de drogas.

\section{MÉTODOS}

Foi realizada uma pesquisa bibliográfica nacional e internacional retrospectiva dos últimos quinze anos utilizando artigos que apresentavam dados e informações relevantes a respeito dos níveis de co-infecção entre HIV e HCV na população em geral e usuários de drogas. Analisou-se aspectos epidemiológicos e fatores de risco para a hepatite C, HIV e co-infecção HCV/HIV, sendo as bases de dados eletrônicas utilizadas SCIELO, LILACS e MEDLINE. Os descritores utilizados foram HIV, hepatite C, co-infecção entre hepatite C e HIV e usuários de drogas.

\section{REVISÃO DA LITERATURA E DISCUSSÃO}

\subsection{O VÍRUS HCV NO BRASIL}

O HCV é transmitido através do contato com sangue e hemoderivados, seja por transfusão sanguínea, hemodiálise, contaminação de dispositivos intravenosos. A via sexual não é tão frequente, sendo importante nos casos de indivíduos que apresentam comportamentos sexuais de risco, destacando-se múltiplos parceiros; sexo sem proteção; traumatismo na relação sexual ou sorologia positiva para DSTs, como sífilis e HIV (AUGUSTO; LOBATO, 2003).

$\mathrm{O}$ vírus HCV apresenta maior viabilidade no meio ambiente quando comparado com o HIV. Dessa forma, é mais efetiva a infecção por HCV por meio da via parenteral, sendo dez vezes mais infectante que o HIV na exposição a materiais perfurocortantes (THORPE et al., 2012). Nesse sentido a toxicodependência de drogas endovenosas é o principal fator de risco para a infecção pelo vírus da hepatite $\mathrm{C}$, uma vez que o $\mathrm{HCV}$ é mais eficientemente transmitido por via parenteral (AUGUSTO; LOBATO, 2003).

Em um estudo realizado em Santos, foi observada uma alta prevalência de hepatite $C$ nesta população, havendo uma incidência de $84,8 \%$. Já para a população não usuária, encontrou-se uma taxa de 20,9\% (SEGURADO et al., 2004).

Segundo um boletim epidemiológico realizado no município de São Paulo, as três principais prováveis fontes/mecanismos de transmissão, de acordo com o banco de dados do Sistema de Informação de Agravos de Notificação (SINAN), são o uso de drogas, sendo o principal meio de aquisição do HCV $(30,4 \%)$, seguido pela via sexual $(26,5 \%)$ e pela transfusional $(20,2 \%)$. Tais resultados confirmam o descrito na literatura em termos de HCV, uma vez que o uso de drogas injetáveis representa a principal via de aquisição da hepatite C. A elevada taxa de transmissão sexual encontrada neste estudo não está de acordo com a literatura atual, sendo provável a explicação para tal fato, a considerável percentagem de fonte de transmissão ignorada $(52,8 \%)$, prejudicando, nesse sentido, a análise desta variável (SÃO PAULO, 2011).

Tabela 1. Número e porcentagem de casos com marcadores para o HCV, segundo a provável fonte/mecanismo de transmissão, município de São Paulo, 2007 a 2010 (continua)

\begin{tabular}{lcc}
\hline Fonte & $\mathbf{N}^{\mathbf{0}}$ de Casos & $\%$ \\
\hline Uso de Droga & 1.691 & 30,4 \\
Sexual & 1.477 & 26,5 \\
Transfusional & 1.124 & 20,2 \\
Tratamento & 485 & 8,7 \\
Cirúrgico & & \\
Tratamento & 288 & 5,2 \\
Odontológico & & 1,2 \\
Transmissão & 69 & 0,9 \\
Vertical & 51 & 0,7 \\
Domiciliar & 38 & \\
$\begin{array}{l}\text { Acidente de } \\
\text { Trabalho }\end{array}$ & & \\
& &
\end{tabular}


(conclusão)

\begin{tabular}{lcc} 
Hemodiálise & 38 & 0,7 \\
Outros & 303 & 5,4 \\
\hline Total & 5.564 & 100 \\
\hline
\end{tabular}

Fonte: COVISA/CCD/SINAN - Hepatites Virais. Retirado do Boletim Epidemiológico de AIDS HIV/DST e Hepatites B e C no município de São Paulo (2011).

Em relação às vias de transmissão, as principais formas identificadas foram o uso de drogas e transfusões sanguíneas. Em 2010, 14,8\% dos casos estavam relacionados com o uso de drogas por parte dos indivíduos estudados e $14,9 \%$ dos casos estavam associados à via transfusional. A co-infecção do HCV e HIV foi registrada em $11,4 \%$ do total de casos confirmados no período de 2007 a 2010 (BRASIL, 2011).

Os usuários de drogas representam um grupo de grande importância dentro do contingente de infectados pelo HCV. Uma amostra desta realidade é demonstrada por meio de um estudo realizado em centros de tratamento de uso de drogas filantrópicos de Goiânia e Campo Grande, no período de agosto de 2005 a julho de 2006. Foi realizado um questionário baseado no instrumento padronizado pela Organização Mundial de Saúde e coletadas amostras de sangue dos usuários, sendo elas submetidas à detecção por ELISA. A média da idade da população estudada foi de 28,3 anos, sendo que $88,3 \%$ deles eram do sexo masculino, $64,3 \%$ solteiros, $59,5 \%$ eram brancos, 70,2\% informaram ter até oito anos de escolaridade e $46,5 \%$ relataram renda familiar entre dois e cinco salários mínimos. Dos 691 pacientes, 49 apresentaram positividade para o marcador anti-HCV, sendo 48 deles confirmados com o ensaio immunoblot, obtendo um valor de 6,9\% de positividade para o antiHCV (LOPES et al., 2009).

Diante do exposto, pode-se afirmar que a hepatite $\mathrm{C}$ acomete principalmente os indivíduos do sexo masculino, que apresentam comportamentos de risco; usuários de drogas ilícitas; indivíduos submetidos a transfusões sanguíneas, sobretudo antes de 1993.

\subsection{O VÍRUS HIV NO BRASIL}

O HIV gera no organismo portador da infecção um processo vírico, que apesar de apresentar fases assintomáticas, caracteriza-se por uma imunodeficiência progressiva, que apresenta enorme capacidade de cronificação e letalidade (OLIVEIRA; SILVESTRE, 2004).

O Brasil foi um dos primeiros países em desenvolvimento a garantir aos indivíduos soropositivos acesso universal e gratuito aos medicamentos antirretrovirais no Sistema Único de Saúde (SUS). No entanto, apesar de existir este amplo acesso desde 1996, o percentual de internações por AIDS no SUS não passou por modificações significativas ao longo do tempo. O que se observou foi um menor número de internações entre pacientes em tratamento com antirretrovirais nas redes de saúde, além do aumento de 2,7 vezes no número de pacientes submetidos à terapêutica adequada entre 1997 e 2003 (DOURADO et al., 2006).

\subsection{CO-INFECÇÃO ENTRE O VÍRUS HIV E HCV}

A co-infecção do vírus HCV em indivíduos portadores do HIV é mais frequentemente observada devido ao fato de ambos os vírus apresentarem similares rotas de infecção, principalmente no que se refere à via parenteral (GABE; LARA, 2008). No grupo dos indivíduos HIV/HCV positivos, destacam-se os usuários de drogas ilícitas injetáveis (BRASIL, 2008).

Nos indivíduos co-infectados a progressão para cirrose hepática ocorre de maneira mais rápida quando comparada com os monoinfectados. Além disso, estudos recentes têm mostrado que o HCV é um cofator importante para uma rápida progressão para a AIDS, acelerando a diminuição do número de linfócitos T CD4 ${ }^{+}$ (BRASIL, 2002).

No Brasil, também se observou um aumento das complicações hepáticas, sendo estimada para o país uma prevalência de co-infecção entre 9,2\% e 54,7\%, dependendo da região geográfica (BRASIL, 2008).

Nesse sentido, a co-infecção entre HIV e HCV passou a gerar números crescentes de morbidade e mortalidade. O vírus HIV altera alguns aspectos da hepatite C, como: aumento da viremia do HCV, elevação das taxas de transmissão vertical, os coinfectados evoluem mais rapidamente para fibrose e insuficiência hepática terminal com incidência aumentada de carcinoma hepatocelular e morte. $\mathrm{O}$ vírus HCV também altera a evolução da infecção 
pelo HIV: aumenta o risco de progressão para a AIDS e morte associada, diminui a resposta ao tratamento por antirretrovirais, e aumenta o risco de toxicidade hepática e metabólica (OLIVEIRA; SILVESTRE, 2004).

No Brasil, em seus primeiros anos de epidemia, aproximadamente $60 \%$ dos casos notificados para o HIV estavam associados a alguma forma de contato sexual. Posteriormente, o HIV disseminou-se entre usuários de drogas injetáveis e pacientes transfundidos. A partir dos anos 90 a infecção aumentou consideravelmente entre 0 sexo feminino, reduzindo a diferença de razão da doença entre os sexos e aumentando a transmissão vertical (ZOCRATTO et al., 2006).

Em um estudo realizado no Hospital Geral de Caxias do Sul com gestantes HIV positivas e seus recém-nascidos, no período entre janeiro de 2000 a dezembro de 2001, foram realizados um total de 3310 nascimentos, sendo que deste total, 47 partos foram de gestantes soropositivas. A idade média das gestantes era de 25,9 anos, sendo que 12,8\% eram adolescentes e 34\% delas eram usuárias de drogas. 70,1\% das 47 gestantes tiveram o diagnóstico de HIV positivo durante a gestação ou parto. Todos os recém-nascidos receberam doses de AZT, sendo que quatro deles receberam leite materno após o nascimento. Dos recém-nascidos acompanhados, apenas dois foram diagnosticados como soropositivos, sendo que suas mães não receberam tratamento com antirretrovirais durante a gestação e no momento do parto, pois o diagnóstico de HIV positivo só foi feito no centro obstétrico após o parto. Dos recém-nascidos que positivaram, uma das mães realizou o pré-natal e a outra era usuária de drogas (STEFANI; ARAUJO; ROCHA, 2004). Tal estudo demonstra o quanto é importante o acompanhamento das gestantes HIV positivas para o controle da transmissão vertical, além de evidenciar a considerável prevalência de usuárias de drogas dentre as infectadas (34\%), comprovando a importância deste grupo no montante dos soropositivos existentes no país.

Os usuários de drogas injetáveis (UDI), desde os anos 80, passaram a ocupar posição de destaque entre os casos de HIV, mantendo sua expansão para determinadas áreas geográficas. A expressiva inclusão dos UDI entre os casos de AIDS foi crucial no processo de expansão da epidemia para municípios de pequeno e médio porte, bem como contribuiu para o aumento dos casos entre mulheres usuárias de drogas injetáveis e entre aquelas que contraíram a doença por meio de relações sexuais com parceiros UDI. Nas regiões Norte e Nordeste encontram-se baixas proporções de casos de HIV adquiridos por meio de drogas injetáveis. Já no Sul e Sudeste do país, a categoria de UDI apresenta grande relevância, com percentuais de infecção semelhantes aos encontrados em casos heterossexuais (BRITO et al., 2007).

A população formada por usuários de drogas injetáveis tem mostrado, nos últimos tempos, ser o grupo onde são crescentes as taxas de infecção por HIV, uma vez que eles estão expostos à via dupla de contaminação (sexual e endovenosa), tendo como agravante para tal situação a alta prevalência de co-infecção com o vírus da hepatite $\mathrm{C}(\mathrm{HCV})$.

Alguns estudos realizados com a população UDI mostram que em locais que adotaram medidas educativas voltadas para os usuários de drogas, houve uma redução dos níveis epidêmicos de transmissão do HIV, revelando que este público é sensível às ações preventivas, sendo capaz de reduzir a frequência de situações de risco. Porém, apesar de se observar uma redução das situações de risco, ainda se constatou níveis significativos de comportamentos de risco neste grupo, mesmo em locais onde tais medidas tiveram um impacto considerável (BRASIL, 2011).

No Brasil, vários estudos revelaram que a droga mais utilizada entre UDI é a cocaína, sendo consumida por 94 a 100\% dos usuários. Os casos de AIDS atribuídos aos UDI estão diretamente relacionados ao tráfico de drogas, principalmente nas regiões de portos marítimos (Santos, Itajaí, São Paulo) e locais que são atravessados pela rota das drogas (Cuiabá, região Sul e Triângulo de Minas Gerais) (CAIAFFA; BASTOS, 1998).

Segundo Brasil (2011), em 2010, do total de 34.218 casos de AIDS notificados, 14.142 (41,3\%) foram notificados na região Sudeste, 7.888 (23,1\%) na região Sul, 6.702 (19,6\%) na região Nordeste, $3.274(9,6 \%)$ na região Norte e 2.211 (6,5\%) na região Centro-Oeste. De forma que os maiores índices de AIDS na região Norte se encontram no Estado do Pará (12.532); no Nordeste estão na Bahia (19.290); no Sudeste se encontram em São Paulo (207.077), no Sul estão no Rio Grande do Sul 
(60.512) e no Centro-Oeste estão em Goiás (12.588).

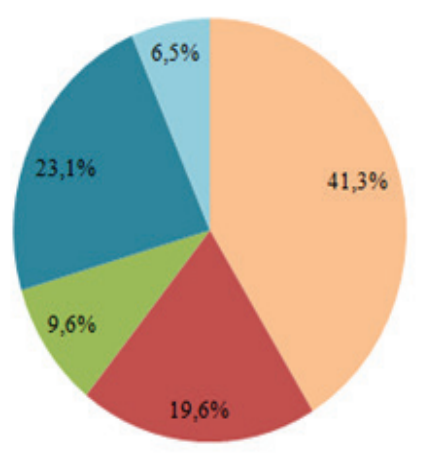

॥ SUDESTE

-NORDESTE

INORTE

$\triangle$ SUL

=CENTRO-OESTE

Figura 1. Distribuição percentual de casos de AIDS por região Fonte: MS/SVS/Departamento de DST, AIDS e Hepatites Virais (2011).

A expansão da AIDS entre os indivíduos de menor escolaridade é um fato que se iniciou na década de 90 , ganhando espaço, sobretudo, na população formada por usuários de drogas injetáveis, heterossexuais e mulheres (FONSECA; SZWARCWALD; BASTOS, 2002).

Diante do que foi exposto, pode-se concluir que a epidemia da AIDS, ao longo do tempo, foi alterando o seu perfil, havendo um aumento do número de casos nas regiões Norte e Nordeste em oposição à queda das taxas nas regiões Sul, Sudeste e Centro-Oeste. Houve também uma diminuição da relação homem/mulher nos casos de AIDS, ocorrendo uma maior expansão da doença em indivíduos heterossexuais e em usuários de drogas injetáveis, além da pauperização da doença, atingindo as camadas com menores recursos financeiros e educacionais.

\subsection{CO-INFECÇÃO ENTRE O HCV E HIV NO BRASIL}

A co-infecção entre estes dois vírus tem ganhado considerável importância no grupo de usuários de drogas injetáveis, uma vez que estes estão expostos constantemente a fatores de risco, como o compartilhamento de agulhas e seringas, além de práticas sexuais sem segurança comuns a esta população.

Um estudo realizado em um grupo de 150 pacientes HIV positivos de Botucatu e região obteve como resultado de prevalência de co-infecção uma percentagem de $14,7 \%$ (CORVINO et al., 2007).
Tabela 2. Número e porcentagem de casos com marcadores para o HCV e informação de presença de coinfecção com HIV/ Aids, segundo a provável fonte/mecanismo de transmissão, município de São Paulo, 2007 a 2010

\begin{tabular}{lcc}
\hline Fonte & $\mathbf{N}^{\mathbf{0}}$ de Casos & $\%$ \\
\hline Uso de Droga & 657 & 49 \\
Sexual & 544 & 40,5 \\
Transfusional & 42 & 3,1 \\
Tratamento & 30 & 2,2 \\
Cirúrgico & & \\
Tratamento & 30 & 2,2 \\
Odontológico & & \\
Transmissão & 10 & 0,7 \\
Vertical & 5 & 0,4 \\
Domiciliar & 3 & 0,2 \\
Hemodiálise & 21 & 1,6 \\
Outros & 1.342 & 100 \\
\hline Total & & \\
\hline
\end{tabular}

Fonte: COVISA/CCD/SINAN- Hepatites Virais. Retirado do Boletim Epidemiológico de AIDS HIV/DST e Hepatites B e C no município de São Paulo (2011).

Outro estudo com usuários de drogas injetáveis, que faz parte do Projeto Ajude-Brasil I, que se trata de um estudo multicêntrico de corte transversal realizado com 287 usuários de drogas injetáveis recrutados do programa de troca de seringas em cinco cidades brasileiras (São Paulo, Sorocaba, São José do Rio Preto, Itajaí e Porto Alegre) obteve uma taxa de co-infecção de 42,6\%, sendo $82,7 \%$ homens e $17,3 \%$ mulheres, com uma média de idade de 29,25 anos. Em termos de raça/cor, igualmente a relatos na literatura, houve um predomínio da cor branca dentre os indivíduos analisados (ZOCRATTO et al., 2006).

Um levantamento sócio-demográfico com usuários de drogas autodeclarados atendidos no Projeto Redução de Danos na Linha, que tem apoio do Programa Nacional de DST, constatou que dos 352 cadastrados até $2003,85 \%$ pertenciam ao sexo masculino, sendo a faixa etária predominante entre 15 a 39 anos. Quanto ao estado civil, verificou-se que $76,70 \%$ se declaravam solteiros. Em termos de escolaridade, a maior parte dos indivíduos (25\%) não possuía o primeiro grau completo, mas a maior percentagem deles encontrava-se empregada $(56,53 \%)$. Dos entrevistados, $1,7 \%$ afirmaram ter sorologia positiva para o HIV e 0,85\% ser HCV positivos (RIO DE JANEIRO, 2003).

A população formada por moradores de rua também representa um grupo com grande vulnerabilidade 
em relação à infecção pelo HIV e HCV. Em uma pesquisa realizada em São Paulo no período de novembro de 2002 a maio de 2003 , foi observado que $3 \%$ dos entrevistados eram usuários de drogas, sendo encontrada uma taxa de co-infecção de $0,6 \%$ para HIV/HCV. A maior parte deles tinha no máximo quatro anos de educação formal (BRITO et al., 2007).

Os indivíduos encarcerados caracterizam-se também como grupo de risco para infecções transmitidas principalmente pelas vias parenterais e sexuais. Estudos realizados em presídios brasileiros revelaram a predisposição desse grupo para adquirir a co-infecção entre HCV e HIV através do uso de drogas injetáveis e de práticas sexuais de risco, além de associarem esse quadro às condições de confinamento, status marginal e serviços de saúde precários. Uma amostra dessa realidade foi obtida a partir de um estudo realizado em um presídio feminino no Rio Grande do Sul, que apresentou como resultados as taxas de $14,47 \%$ para o $\mathrm{HCV}, 9,21 \%$ para o HIV e 2,36\% para a co-infecção HIV/HCV (GABE; LARA, 2008).

No Brasil, os níveis de co-infecção variam de acordo com as diferentes regiões, sendo encontradas as maiores taxas nas regiões Sul e Sudeste do país, pois são nestas em que se encontra a maior frequência do consumo de drogas injetáveis dentre a população, situação divergente da encontrada no Nordeste (CARVALHO et al., 2009). Um estudo realizado no hospital universitário de Recife, no período de março a dezembro de 2003, obteve como resultado de prevalência a taxa de 4,1\% (CARVALHO et al., 2009). Já outro estudo realizado na cidade de Maceió, obteve-se uma prevalência de 3,3\% (SANTOS, 2010). Tais resultados foram considerados abaixo do padrão nacional. Em ambos os estudos, houve um predomínio do sexo masculino entre os coinfectados, sendo tal fato semelhante ao que se relata na literatura.

Um estudo visando avaliar a epidemiologia de pacientes coinfectados HIV/HCV atendidos na Fundação Santa Casa de Misericórdia do Estado do Pará analisou 31 pacientes no período de agosto de 2004 a agosto de 2005. Dos resultados obtidos, observou-se uma predominância do sexo masculino $(80,6 \%)$, sendo que $29 \%$ deles se encontravam na faixa etária entre 43 e 49 anos e 71\% eram solteiros. Tais achados estão relacionados com o fato de a população masculina, principalmente os indivíduos solteiros, se expor a fatores de risco. Dos participantes estudados, $32,3 \%$ deles relataram ter história de transfusões de sangue, sendo que desse valor, 22,6\% receberam tais transfusões antes de 1993. Em relação ao comportamento sexual, observou-se que $61,3 \%$ dos entrevistados utilizam preservativos às vezes; 58,1\% deles apresentam de 2 a 5 parceiros, sendo 64,5\% heterossexuais. Quanto ao uso de drogas, 54,8\% relataram fazer uso de alguma droga injetável (AMARAL et al., 2007).

Outro estudo realizado na mesma instituição, no período de agosto de 2004 a abril de 2008, constatou que dos 62 pacientes coinfectados por HCV/ HIV $79 \%$ pertenciam ao sexo masculino, 59,7\% eram heterossexuais, $67,74 \%$ apresentavam o estado civil solteiro e 48,4\% afirmaram fazer uso de drogas ilícitas injetáveis (AMARAL et al., 2010). A Tabela 43 abaixo mostra as prevalências dos estudos anteriormente abordados em diferentes regiões do país.

Tabela 3. Taxa de co-infecção em diferentes regiões

\begin{tabular}{lcc}
\hline Cidade/Estado & Região & $\begin{array}{c}\text { Taxas de co- } \\
\text { infecção }\end{array}$ \\
\hline $\begin{array}{l}\text { Botucatu (CORVINO et al., } \\
\begin{array}{l}\text { 2007) } \\
\text { Rio Grande do Sul (TOVO et } \\
\text { al., 2006) }\end{array}\end{array}$ & SUDESTE & $14,70 \%$ \\
$\begin{array}{l}\text { São Paulo (MARCHESINI et } \\
\text { l., 2007) }\end{array}$ & SUDESTE & $84 \%$ \\
$\begin{array}{l}\text { Recife (CARVALHO et al., } \\
\text { 2009) }\end{array}$ & NORDESTE & $4,10 \%$ \\
$\begin{array}{l}\text { Maceió (SANTOS et al., } \\
\text { 2009) }\end{array}$ & NORDESTE & $3,30 \%$ \\
\hline
\end{tabular}

Fonte: Autor (2013).

Em relação à população atingida, observa-se que os usuários de drogas (Tabela 4) são o principal grupo dentre os coinfectados, sendo esta evidência também visualizada nos estudos anteriormente mencionados. 
Tabela 4. Taxas de usuários de droga entre os co-infectados

\begin{tabular}{lc}
\hline Autores & Usuários de drogas \\
\hline (TOVO et al., 2006) & $75,3 \%$ \\
(BINELLI et al., 2012) & $59 \%$ \\
(MARCHESINI et al., 2007) & $57 \%$ \\
(AMARAL et al., 2007) & $54,8 \%$ \\
(AMARAL et al., 2010) & $48,4 \%$ \\
\hline
\end{tabular}

Fonte: Autor (2013).

Os usuários de drogas, como o principal grupo envolvido na co-infecção HIV/HCV, é um grupo formado, em geral, por indivíduos pertencentes ao sexo masculino, com baixa escolaridade, sem trabalho fixo e praticante de formas inseguras de relações sexuais.

Em termos de fatores de risco para a co-infecção, observa-se que em indivíduos do sexo masculino, tais fatores estão mais associados ao uso de drogas injetáveis e o consequente hábito de compartilhamento de seringas e agulhas. Já no sexo feminino, apesar das taxas de coinfecção serem menores neste grupo, percebe-se que, quando ocorre, se deve também ao uso de drogas e às relações heterossexuais, uma vez que é comum dentre os UDI a prática de relações sexuais sem proteção e com múltiplos parceiros (SILVA; BARONE, 2006).

\section{CONCLUSÃO}

Com base na revisão apresentada, observou-se que a hepatite Cé um fator de risco para morbimortalidade em portadores de HIV/AIDS em todo o mundo. Observouse que a população de usuários de drogas representa o grupo de maior risco para a aquisição da co-infecção HIV/ $\mathrm{HCV}$, tendo ela características de expansão semelhantes às da epidemia da AIDS, uma vez que vem atingindo consideravelmente os indivíduos com menos recursos financeiros e educacionais. Devido à importância da coinfecção HIV/HCV, torna-se fundamental o conhecimento da epidemiologia destas doenças. As grandes variações regionais e a existência de poucos dados sobre essa realidade no Brasil elevam a necessidade de dados representativos desta realidade, a fim de permitir o tratamento destas patologias e adequar o planejamento dos serviços de saúde.

\section{REFERÊNCIAS}

AMARAL, I. S. A. et al. Epidemiology of HIV/HCV coinfected patients attended at Fundação Santa Casa de Misericórdia do Pará. Rev. Para. Med., Belém, v. 21, n. 1, mar. 2007.

AMARAL, I. S. A. et al. Coinfection caused by the human immunodeficiency virus and hepatitis $\mathrm{C}$ virus (HIV-1/ HCV): a case study in the Brazilian Amazon. Rev PanAmaz Saude, Ananindeua, v. 1, n. 2, jun. 2010.

AUGUSTO, F.; LOBATO, C. Hepatite C. Hepatites Víricas (J. Cottered), p. 99-122, 2003.

BARTLET, J. G. The Johns Hopkins Hospital 2002 Guide to Medical Care of Patients with HIV Infection. Philadelphia: Lippincott William and Wilkis, 2002. p. 1405. 2002.

BRASIL. Ministério da Saúde. Secretaria de Vigilância em Saúde. Boletim epidemiológico-Hepatites virais. Brasília (DF): [s.n.], 2011.

BRASIL. Ministério da Saúde. Secretaria de Vigilância em Saúde. O Brasil está atento: Hepatites Virais. Brasília (DF): [s.n.], 2008.

BRASIL. Ministério da Saúde. Secretaria de Políticas de Saúde. Programa Nacional de Hepatites Virais. Recomendações para Tratamento da Co-Infecção entre HIV e Hepatites Virais. Brasília (DF): [s.n.], 2002.

BRITO, V. O. C. et al. Infecção pelo HIV, hepatites B e C e sífilis em moradores de rua. Rev. Saúde Pública, v. 41, n. Supl 2, p. 47-56, 2007.

CAIAFFA, W. T. et al. Usuários de drogas injetáveis e infecção pelo vírus da imunodeficiência humana: epidemiologia e perspectivas de intervenção. Rev Bras Epidemiol, v. 1, n. 2, p. 190-202, 1998.

CARVALHO, F. H. P. et al. Co-infecção por HIV/HCV em hospital universitário de Recife, Brasil. Rev Saúde Pública, v. 43, n. 1, p. 133-39, 2009.

CORVINO, S. M. et al. Co-infecção HIV/HCV em pacientes de Botucatu e região. Rev Bras Epidemiol, p. 537-543, 2007. 
DOURADO, I. et al. Tendências da epidemia de Aids no Brasil após a terapia anti-retroviral. Rev Saúde Pública, v. 40, n. supl., p. 9-17, 2006.

FONSECA, M. G. P.; SZWARCWALD, C. L.; BASTOS, F. I. Análise sociodemográfica da epidemia de Aids no Brasil, 1989-1997. Rev Saúde Pública, v. 36, n. 6, p. 678-85, 2002.

GABE, C.; LARA, G. M. Prevalência de anti-HCV, anti-HIV e co-infecção HCV/HIV em um presídio feminino do Estado do Rio Grande do Sul. RBAC, v. 40, n. 2, p. 87-89, 2008.

LOPES, C. L. R. et al. Prevalence, risk factors and genotypes of hepatitis $C$ virus infection among drug users, CentralWestern Brazil. Rev Saúde Pública, v. 43, p. 43-50, 2009.

OLIVEIRA, J.; SILVESTRE, A. M. História natural da Infecção pelo HIV. Departamento de Doenças Infecciosas dos Hospitais da Universidade de Coimbra. In: CONGRESSO VIRTUAL HIV/AIDS, 3., 2002, Coimbra. Anais... Coimbra, PT, 2002.

RIO DE JANEIRO. Secretaria Estadual de Saúde. Co infecção HIV e Hepatites Virais em usuários de drogas e seus parceiros(as) clientes de um programa de Redução de Danos. Núcleo do Estado do Rio de Janeiro da Rede Nacional de Pessoas Vivendo Com HIV/ AIDS. 2003.

ROSS, M. W.; WILLIAMS, M. L. Sexual behavior and illicit drug use. Annual Review of Sex Research, v. 12, n. 1, p. 290-310, 2001.

SANTOS, K. F. et al. Alterações laboratoriais encontradas em indivíduos co-infectados pelo vírus da imunodeficiência humana (HIV) e pelo vírus da hepatite C (HCV). RBAC, v. 42, n. 1, p. 21-24, 2010.

São Paulo. Secretaria Municipal de Saúde. Boletim Epidemiológico de AIDS HIV/DST e Hepatites B e C no município de São Paulo. Secretaria Municipal de Saúde. São Paulo (SP); 2011.

SEGURADO, A. C. et al. Hepatitis $C$ virus coinfection in a cohort of HIV-infected individuals from Santos, Brazil: seroprevalence and associated factors. AIDS patient care and STDs, v. 18, n. 3, p. 135-143, 2004.

SILVA, A. C. M.; BARONE, A. A. Fatores de risco para infecção pelo HIV em pacientes com o vírus da hepatite C. Rev Saúde Pública, v. 40, n. 3, p. 482-8, 2006.

STEFANI, M.; ARAÚJO, B. F.; ROCHA, N. M. P. Transmissão vertical do HIV em população de baixa renda do Sul do Brasil. DST. J bras Doenças Sex Transm, v. 16, n. 2, p. 33-39, 2004.

THORPE, L. E. et al. Risk of hepatitis C virus infection among young adult injection drug users who share injection equipment. Am J Epidemiol., v. 155, n. 7, p. 645-653, 2002.

ZOCRATTO, K. B. F. et al. HCV and HIV infection and coinfection: injecting drug use and sexual behavior, AjUDEBrasil I Project. Cad Saúde Pública, v. 22, n. 4, p. 839848, 2006.

Recebido em: 18 de novembro de 2014 Aceito com modificações: 01 de abril de 2015 